\title{
MicroRNAs that respond to histone deacetylase inhibitor SAHA and p53 in HCT116 human colon carcinoma cells
}

\author{
SANGSU SHIN ${ }^{1 *}$, EUN-MEE LEE $^{1 *}$, HWA JUN CHA ${ }^{1}$, SEUNGHEE BAE $^{1}$, JIN HYUK JUNG $^{1}$, \\ SUN-MI LEE ${ }^{1}$, YOUNGMIN YOON ${ }^{1}$, HYUNJIN LEE ${ }^{1}$, SUMI KIM ${ }^{1}$, HYUNSOOK KIM ${ }^{1}$, \\ SU-JAE LEE ${ }^{2}$, IN-CHUL PARK ${ }^{3}$, YOUNG-WOO JIN ${ }^{4}$ and SUNGKWAN AN ${ }^{1,5}$ \\ ${ }^{1}$ Functional Genoproteome Research Centre, Konkuk University, Seoul 143-701; ${ }^{2}$ Department of Chemistry, \\ Hanyang University, Seoul 133-791; ${ }^{3}$ Laboratory of Functional Genomics, Korea Institute of Radiological \\ and Medical Sciences, Seoul 139-706; ${ }^{4}$ Division of Radiation Effect Research, Radiation Health \\ Research Institute of KHNP, Seoul 132-703; ${ }^{5}$ LIFEnGENE, Inc., Seoul 143-701, Korea
}

Received May 4, 2009; Accepted July 9, 2009

DOI: 10.3892/ijo_00000452

\begin{abstract}
MicroRNAs (miRNAs) are important post-transcriptional regulators involved in many biological processes. We investigated the expression profiles of miRNAs affected by the histone deacetylase inhibitor, suberoylanilide hydroxamic acid (SAHA), and p53 in the human colon cancer cell line, HCT116 (wt-p53) and its derivative, HCT116 (null-p53). In a microarray assay, 144 of 275 human miRNAs showed several-fold changes in transcription. Most of these miRNAs were strongly affected by SAHA, and their expression profiles varied depending on the presence of p53. Thirty-one miRNAs showing the greatest expression changes were selected for target prediction, and genes related to apoptosis (102), cell cycle (38), and differentiation (102) were predicted. Each miRNA had many target genes, and several genes also were targeted by many miRNAs. Putative p53 upstream binding sites for the miRNAs were determined, and most sites scored $>85 \%$, suggesting a high probability of binding. In conclusion, we identified several miRNAs whose expression was affected by both SAHA and p53. Many of the miRNAs showed dramatic changes and were predicted to target many mRNAs. Further studies will be needed to verify these predictions.
\end{abstract}

\section{Introduction}

Histone acetylation is an important epigenetic modification of chromatin that regulates gene expression and genome

Correspondence to: Dr Sungkwan An, Functional Genoproteome Research Centre, Konkuk University, 1 Hwayang-dong, Gwangjin-gu, Seoul 143-701, Korea

E-mail: ansfgrc@konkuk.ac.kr

${ }^{*}$ Contributed equally

Key words: microRNA, histone deacetylase inhibitor, SAHA, p53, HCT116 stabilization $(1,2)$. The acetylation status of histones is regulated and balanced during DNA replication, cell cycle progression, transcription, and post-transcriptional events by the catalytic activity of histone acetylases (HATs) and histone deacetylases (HDACs) (3). Therefore, these two enzymes are important in regulating gene expression, and their perturbation could play a role in tumorigenesis by bringing about aberrant gene expression (4-6). It was found that HATs and HDACs are involved in the acetylation of histones, as well as in other proteins involved in diverse cellular processes, such as transcription and translation, DNA repair, recombination and replication, signaling, apoptosis, and metabolism (7).

Recently, HDAC inhibitors (HDACIs) were identified as anticancer agents (8-10). These compounds cause differentiation, growth arrest, and apoptosis of transformed cells both in vitro and in vivo, whereas normal cells are relatively less affected (11-16). There are four chemical groups of HDACIs: hydroxamic acid derivatives, cyclic peptides, short-chain aliphatic acids, and benzamides, which are known to inhibit class I, II, and IV HDACs (9). HATs and HDACs regulate diverse cellular processes, and it is known that several transcription factors, such as p53, KLF5, GCMa, GATA-1, TFIIE, TFIIF, and TFII-I, are regulated by HDACs (17-22). Thus, HDACIs can modulate expression of genes, directly or indirectly, through modification of histones and transcription factors (23).

miRNAs are single-stranded, small RNA fragments transcribed primarily by RNA polymerase II and trimmed by the microprocessor complex and Dicer (24-28). They are included in the miRNA-induced silencing complex (miRISC) and are involved in the regulation of gene expression posttranscriptionally by binding to the 3 ' UTR of mRNAs $(28,29)$. miRNAs have been shown to play a role in many biological events, such as development, differentiation, cell cycle, apoptosis, stemness, and tumorigenesis (30-33). Importantly, it has been shown that many types of cancers are induced by aberrant miRNA expression and that are classified by miRNA profiles $(34,35)$. 
In this study, we identified miRNA expression profiles induced by SAHA in human colon carcinoma cell lines which, coincidently, were affected by p53. SAHA is a hydroxamic acid derivative of the HDACI group, which could act as a cancer therapeutic by arresting cancer cell growth or inducing apoptosis $(9,16,36)$. We predicted the targets of several SAHA-induced miRNAs and identified putative p53 binding sites for several miRNAs.

\section{Materials and methods}

Cell culture. The human colon carcinoma cell line, HCT116 (wt-p53) and its p53-null derivative HCT116 (null-p53), were cultured in McCoy's 5A medium containing 10\% fetal bovine serum (FBS) and antibiotics, at $37^{\circ} \mathrm{C}$ in a humidified chamber supplemented with $5 \% \mathrm{CO}_{2}$. One day before irradiation, $5 \times 10^{5}$ cells were seeded in $60-\mathrm{mm}$ culture dishes.

SAHA treatment and RNA preparation. Cells cultured for $24 \mathrm{~h}$ were treated with 0 or $1.5 \mu \mathrm{M}$ SAHA. The SAHA-treated cells were maintained for $24 \mathrm{~h}$ before extracting total RNA. Total RNA was extracted with TRIzol ${ }^{\circledR}$ reagent (Invitrogen, CA, USA) according to the manufacturer's protocol. For the microarray studies, the quality and concentration of RNA samples were determined using an Agilent 2100 Bioanalyzer (Agilent Technologies, CA, USA) and an Ultrospec 3300 Pro UV/Visible Spectrophotometer (Amersham Biosciences, NJ, USA).

Microarray analysis of miRNA profiles. A human miRNA microarray kit (version 2, Agilent Technologies), which contained probes for 723 human and 76 human viral miRNAs, was adapted to analyze the expression profiles of miRNAs. Before hybridizing the miRNAs to probes on the microarray, total RNA (100 ng), including miRNAs, was dephosphorylated by calf intestine alkaline phosphatase (CIP) and denatured by adding dimethyl sulfoxide (DMSO) followed by heating. The dephosphorylated RNA was labeled with pCp-Cy3 using T4 RNA ligase, and the labeled RNA was purified using a Micro Bio-Spin P-6 column (Bio-Rad Laboratories, CA, USA). The purified RNA was denatured and hybridized to the microarray at $55^{\circ} \mathrm{C}$, with shaking at $20 \mathrm{rpm}$ for $20 \mathrm{~h}$ in the Agilent Microarray Hybridization Chamber (Agilent Technologies). The microarray slide was washed and scanned to obtain a microarray image using an Agilent scanner. The numerical data for the miRNA profiles were extracted from the image by a Feature Extraction program (Agilent Technologies) and then were analyzed using GeneSpring GX software, version 7.3 (Agilent Technologies).

Selection of miRNAs. The 723 human miRNAs on the microarray were selected for further analysis. miRNAs with expression changes in at least one sample were filtered and applied to the fold-change analysis. The fold-change analysis was conducted by looking for $>2$-fold changes between four groups: HCT116 (wt-p53) control, SAHAtreated HCT116 (wt-p53), HCT116 (null-p53) control, and SAHA-treated HCT116 (null-p53). miRNAs showing these changes were selected. Of these, miRNAs showing changes $>5$-fold between groups and having expression levels $>5$-fold different at least once were selected for target prediction analysis.

Target prediction of miRNAs. The candidate miRNAs affected by SAHA and/or p53 were chosen for target prediction and analyzed using two target prediction tools, miRBase Targets Version 5 in the Enright Lab at the Wellcome Trust Sanger Institute website (http://microrna.sanger.ac.uk/targets/v5/) and the Gene Ontology website (http://www.geneontology. org/). Human genes related to apoptosis, cell cycle, and differentiation, and proven experimentally, were selected from the latter. Finally, genes predicted as targets of candidate miRNAs and selected from the Gene Ontology were aligned, and matched.

Analysis of p53 binding sites upstream of miRNAs. Fifty kilobases $(\mathrm{kb})$ of upstream sequence from differentially expressed miRNAs (regardless of p53 status) were obtained using the UCSC human BLAT search (http://genome. ucsc.edu/cgi-bin/hgBlat). Putative p53 binding sites upstream of the miRNA sequences were predicted and scored using the p53MH program (37). The top three binding sites were selected and listed in Table III.

\section{Results and Discussion}

This study was aimed at identifying miRNA profiles affected by SAHA and p53 in two HCT116 human colon carcinoma cell lines, one with and one without p53. After treating both cell lines with SAHA for $24 \mathrm{~h}$, total RNA was extracted from both treated and control samples and processed for microarray analysis. Of the 723 human miRNAs selected for study, 275 showed meaningful changes in their transcription profiles. Fig. 2A shows their expression profiles, represented by colors. Changes in expression ranged from $10^{-2}$ to $10^{3}$. miRNAs showing changes in expression level in the presence of SAHA and p53 were sorted by fold-change analysis.

miRNAs showing $>2$-fold change by SAHA or p53 are described in Fig. 1B. Of the 275 human miRNAs studied, 144 showed a $>2$-fold change, indicating that $52.5 \%$ of miRNAs were changed by SAHA or p53. The Venn diagram in Fig. 2A shows a more detailed comparison of the miRNA profiles among the four groups, D0: HCT116 (wt-p53) vs. (null-p53), no SAHA treatment, DW: HCT116 (wt-p53), no SAHA vs. $1.5 \mu \mathrm{M}$ SAHA treatment, DN: HCT116 (null-p53), no SAHA vs. $1.5 \mu \mathrm{M}$ SAHA treatment, and D1.5: HCT116 (wt-p53) vs. (null-p53) with $1.5 \mu \mathrm{M}$ SAHA treatment. Forty miRNAs showed different expression levels as a function of p53 alone, and all but one (143 miRNAs) showed miRNA changes induced by SAHA in both HCT116 (wt-p53) and HCT116 (null-p53) cells. Treatment of HCT116 (wt-p53) and HCT116 (null-p53) cells with $1.5 \mu \mathrm{M}$ SAHA resulted in 51 (DW) and 123 (DN) miRNAs that were changed, respectively. Thirty-one of these miRNAs were common to both DW and DN samples. Of 41 miRNAs expressed differently in HCT116 (wt-p53) and HCT116 (null-p53), 28 showed altered expression levels after SAHA addition. Fifty miRNAs which were expressed at similar levels in untreated HCT116 (wt-p53) and HCT116 (null-p53) cells 
$\mathbf{A}$

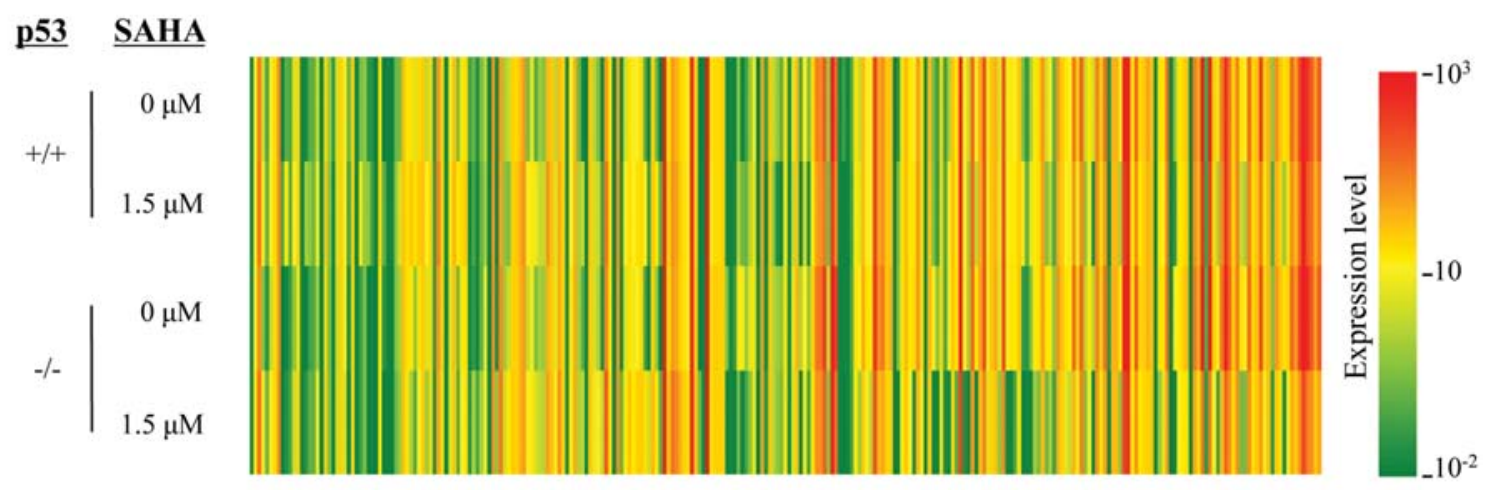

B

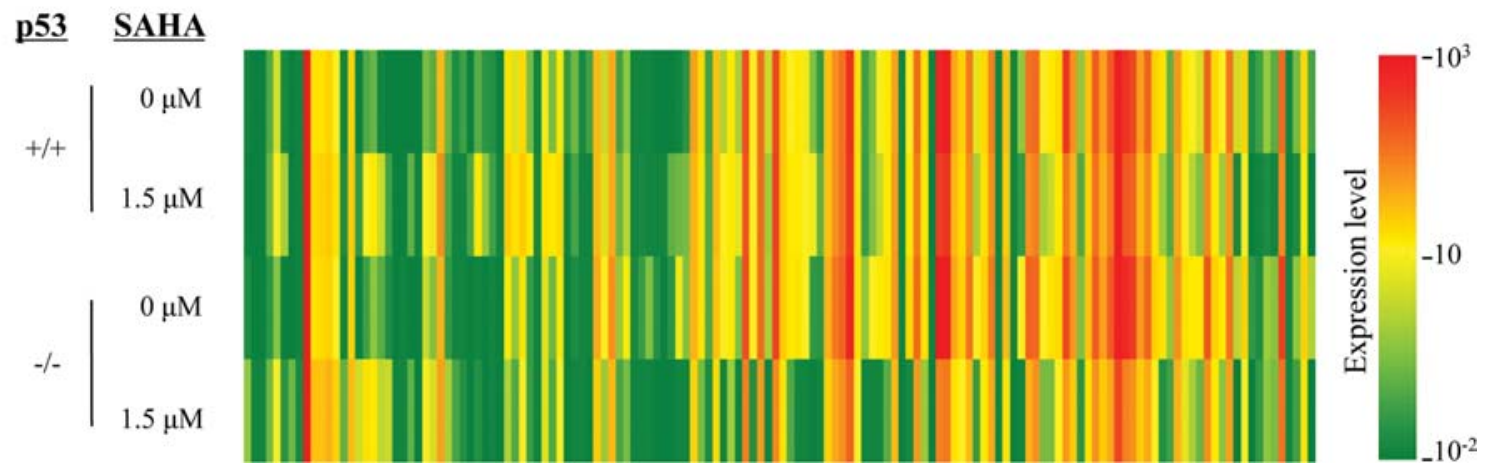

Figure 1. Expression profiles of miRNAs. A total 275 miRNAs were selected by filtering the microarray data, and their expression levels are represented by colors (A). From the identified miRNAs, fold-change analysis was conducted, and miRNAs with expression changed $>2$-fold are marked by a color range (B).

A

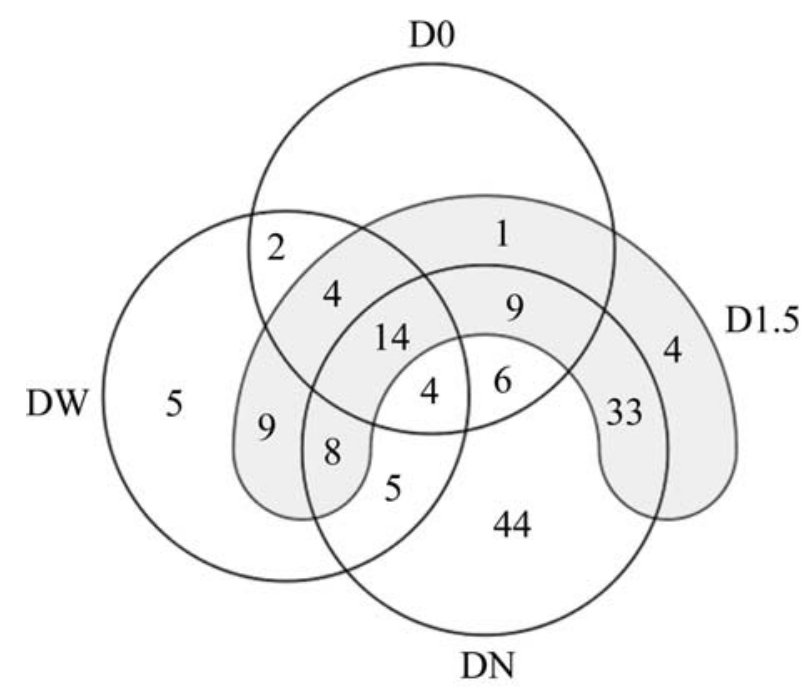

B

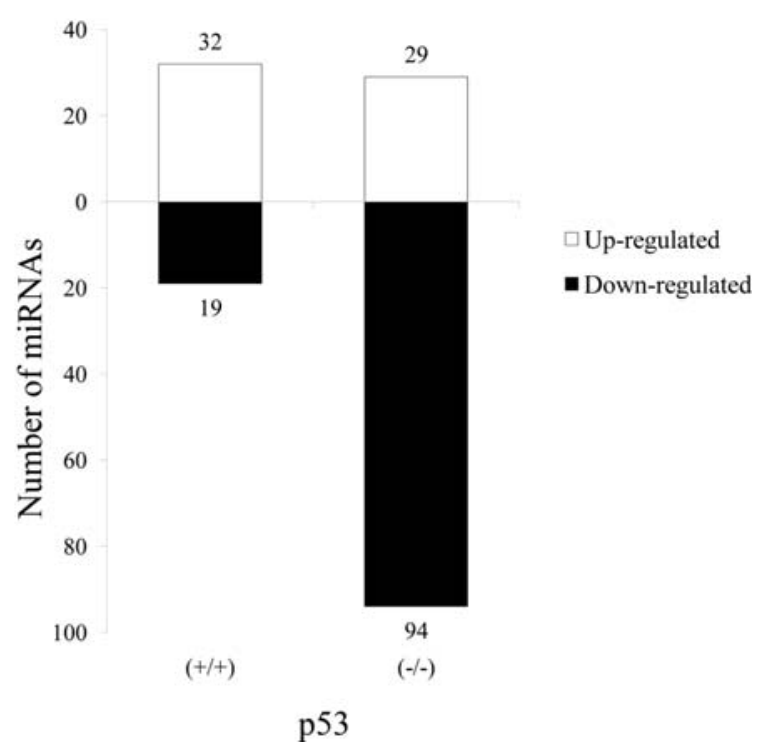

Figure 2. The distribution and the patterns of expression of the miRNAs changed $>2$-fold between each group. The fold-change analysis was conducted between wt-p53 and null-p53 in $0 \mu \mathrm{M}$ SAHA (D0), wt-p53 and null-p53 in $1.5 \mu \mathrm{M}$ SAHA (D1.5), wt-p53 in 0 and $1.5 \mu \mathrm{M} \mathrm{SAHA} \mathrm{(DW),} \mathrm{and} \mathrm{null-p53} \mathrm{in} 0$ and $1.5 \mu \mathrm{M}$ SAHA (DN). For those miRNAs showing $>2$-fold change, the distribution and overlap for all groups are described in a Venn diagram (A). The numbers of miRNAs up- or down-regulated by SAHA treatment in each wt-p53 and null-p53 sample are also described (B).

were changed by SAHA treatment. However, 54 miRNAs present in similar amounts in untreated HCT116 (wt-p53) and HCT116 (null-p53) remained unchanged upon SAHA treatment. One could conclude that these miRNAs were 
Table I. miRNAs that changed $>5$-fold between groups and showed expression levels $>5$-fold change in at least one experiment.

Fold changes

\begin{tabular}{|c|c|c|c|c|}
\hline Name & $\begin{array}{c}\text { D0 (wt-p53 vs. } \\
\text { null-p53) }\end{array}$ & $\begin{array}{l}\mathrm{DW} \text { (SAHA } 0 \text { vs. } \\
1.5 \text { in wt-p53) }\end{array}$ & $\begin{array}{l}\text { DN (SAHA } 0 \text { vs. } \\
1.5 \text { in null-p53) }\end{array}$ & $\begin{array}{l}\text { D1.5 (wt-p53 vs. null-p53 } \\
\text { in SAHA .15) }\end{array}$ \\
\hline hsa-miR-7-1 & 2.44 & 1.06 & -30.54 & -13.28 \\
\hline hsa-miR-9 & 1.20 & -1.36 & -5.00 & -3.07 \\
\hline hsa-miR-22 & 15.46 & 34.90 & 2.14 & -1.06 \\
\hline hsa-miR-29b-1 & 1.04 & -3.61 & -19.33 & -5.12 \\
\hline hsa-miR-30c & -1.04 & -1.15 & -9.85 & -8.94 \\
\hline hsa-miR-32 & 1.17 & -43.11 & 1.21 & -41.50 \\
\hline hsa-miR-100 & -1.11 & -16.56 & -47.83 & -3.22 \\
\hline hsa-miR-132 & 3.20 & 2.88 & -19.33 & -17.42 \\
\hline hsa-miR-141 & 1.90 & -2.96 & -56.81 & -10.09 \\
\hline hsa-miR-153 & 1.07 & 1.05 & -39.92 & -39.14 \\
\hline hsa-miR-194 & -18.06 & 9.62 & 42.12 & -4.13 \\
\hline hsa-miR-196a & 1.32 & 1.11 & -6.06 & -5.11 \\
\hline hsa-miR-212 & 1.17 & -5.16 & -2.14 & 2.81 \\
\hline hsa-miR-221 & 25.62 & 11.70 & -24.05 & -10.99 \\
\hline hsa-miR-222 & 2.48 & -132.71 & -41.09 & 8.00 \\
\hline hsa-miR-362-3p & 1.47 & 1.70 & -23.98 & -27.64 \\
\hline hsa-miR-373 & -2.55 & 1.91 & -20.19 & -98.64 \\
\hline hsa-miR-374b & 1.39 & -1.36 & -46.23 & -24.32 \\
\hline hsa-miR-424 & -1.31 & 3.74 & 9.38 & 1.91 \\
\hline hsa-miR-449a & -1.33 & 1.39 & -13.43 & -24.71 \\
\hline hsa-miR-487b & 1.09 & -5.59 & -1.15 & 5.32 \\
\hline hsa-miR-508-3p & 2.37 & -1.06 & -21.49 & -8.59 \\
\hline hsa-miR-520d-3p & -12.10 & 4.16 & 3.95 & -12.72 \\
\hline hsa-miR-526b & -1.05 & 11.92 & 57.05 & 4.54 \\
\hline hsa-miR-548c-5p & 3.83 & -1.28 & 7.18 & 35.23 \\
\hline hsa-miR-557 & 10.26 & 27.99 & 9.11 & 3.34 \\
\hline hsa-miR-593 & -11.10 & 3.98 & 1.11 & -39.86 \\
\hline hsa-miR-625 & 2.14 & 1.09 & -52.41 & -26.73 \\
\hline hsa-miR-652 & -1.15 & -1.22 & -29.55 & -27.98 \\
\hline hsa-miR-877 & 19.51 & 1.45 & -17.35 & -1.29 \\
\hline hsa-miR-921 & 1.56 & -9.84 & -1.17 & 13.03 \\
\hline
\end{tabular}

affected by SAHA but not by p53. Thus, SAHA appears to affect the expression of many kinds of miRNAs.

The number of miRNAs up- or down-regulated by SAHA in HCT116 (wt-p53) and HCT116 (null-p53) is described in Fig. 2B. There were 32 miRNAs up-regulated in HCT116 (wt-p53) and 29 up-regulated in HCT116 (null-p53) after the addition of SAHA. HCT116 (null-p53) cells showed nearly $>5$-fold down-regulated miRNAs than HCT116 (wt-p53) in the presence of SAHA. This implies that p53 may alter the expression of certain miRNAs when the cells are treated with SAHA due to its effect on transcription factors or regulator expression.
Table I lists those miRNAs that showed a $>5$-fold change between any group and exhibited a $>5$-fold change in expression level in at least one experiment. There were 31 miRNAs that met this criteria and, interestingly, many asterisked miRNAs, miR-7-1*, $-22^{*},-29 b-1^{*},-100^{*},-141^{*},-221^{*},-222^{*}$, $-424^{*}$, and $-877^{*}$, often detected at very low levels as biogenesis byproducts that likely are non-functional, were included (38). These miRNAs showed high levels of relative change, although most of their dominant miRNAs did not show much change and some dominant miRNAs were changed only 2- to 3 -fold by SAHA or p53 (data not shown). Although further studies are needed to confirm these data, it could be that the 
Table II. miRNAs and their predicted target genes associated with apoptosis, cell cycle, and differentiation.

\begin{tabular}{|c|c|c|c|}
\hline \multirow[b]{2}{*}{ miRNA } & \multicolumn{3}{|c|}{ Biological functions and related genes } \\
\hline & Apoptosis & Cell cycle & Differentiation \\
\hline $\operatorname{miR}-7-1^{*}$ & $\begin{array}{l}\text { AVEN, CDKN2C, GDNF, NRG1, } \\
\text { TERF1 }\end{array}$ & $\begin{array}{l}\text { APC, BTG3, CDKN2C, } \\
\text { CDKN2D, DBC1, EGF, } \\
\text { MLF1, SPHK1, ZWINT }\end{array}$ & $\begin{array}{l}\text { ATP2B2, CALCA, GDNF, NCOA6, NRG1, } \\
\text { ROBO1 }\end{array}$ \\
\hline $\operatorname{miR}-9$ & $\begin{array}{l}\text { BNIP3, CDKN2A CIDEC, DEDD2, } \\
\text { IL12A, PCSK6, PPP3R1, SRGN }\end{array}$ & CDKN2A, IL12A, WDR6 & APOB, CD36, NP \\
\hline $\operatorname{miR}-22^{*}$ & $\begin{array}{l}\text { AIFM3, CASP8, CIDEC, FASLG, } \\
\text { IL6, RTKN, TP53BP2, TRADD }\end{array}$ & ZW10 & $\begin{array}{l}\text { APOB, AZU1, CALCA, CAV3, CSF2, } \\
\text { DHRS2, EIF2B2, EIF2B5, FARP2, IL6, } \\
\text { MAPK12, RTN4, SCEL, SPRR1A, SPRR1B }\end{array}$ \\
\hline $\operatorname{miR}-29 b-1^{*}$ & $\begin{array}{l}\text { CECR2, DFFB, ING4, KIAA1967, } \\
\text { LGALS12, NLRC4, NUAK2, SCG2 }\end{array}$ & ING4, MLF1, NEK6 & $\begin{array}{l}\text { ANXA1, CHRNB1, CSF2, ENG, NF2, } \\
\text { OBSL1, SCIN, SPI1, TNFSF11, ZAP70 }\end{array}$ \\
\hline miR-30c & $\begin{array}{l}\text { AVEN, DFFB, GCLC, P2RX4, } \\
\text { RASA1, SON, TCF7L2 }\end{array}$ & GTPBP4 & $\begin{array}{l}\text { ACTA1, ACVR1, ADAM9, ATP2B2, } \\
\text { DOCK7, GLI2, IQCB1, JAG2, ROBO2, } \\
\text { RPS19, SCEL, TCF7L2, TGM3 }\end{array}$ \\
\hline $\operatorname{miR}-32$ & BRCA1, HBXIP & - & $\begin{array}{l}\text { BMPR2, CALCA, CHD7, GPR98, IL6ST, } \\
\text { NCOA6, NKX2-5, TCFL5, TTN }\end{array}$ \\
\hline $\operatorname{miR}-100^{*}$ & $\begin{array}{l}\text { CASP8, GHRL, GRIK2, P2RX4, } \\
\text { RYR2, TCF7L2 }\end{array}$ & NEK11, NUSAP1 & ANXA1, GHRL, RUNX1, SCEL, TCF7L2 \\
\hline miR-132 & $\begin{array}{l}\text { CASP8, GHRL, HIP1, IL6, LCK, } \\
\text { PEA15, PML, RASA1, YWHAB }\end{array}$ & $\begin{array}{l}\text { APC, ASNS, GMNN, } \\
\text { PML }\end{array}$ & $\begin{array}{l}\text { ACVR1, ACVR2B, CYFIP1, DOCK7, } \\
\text { ELF3, FARP2, GHRL, IL6, LCK, SART1, } \\
\text { TGM3, TNP1, UPK1B, ZAP70 }\end{array}$ \\
\hline $\operatorname{miR}-141^{*}$ & $\begin{array}{l}\text { DLC1, ERCC3, LCK, NLRP1, } \\
\text { TNFRSF10B }\end{array}$ & $\begin{array}{l}\text { AFAP1L2, ALOX15B, } \\
\text { ZW10 }\end{array}$ & $\begin{array}{l}\text { CHRNA1, CNTN4, CRP, EIF2B3, ERCC3, } \\
\text { GPR98, IL27, IL31RA, LCK, ROBO2 }\end{array}$ \\
\hline miR-153 & $\begin{array}{l}\text { AKT1, BAD, BRCA1, CASP10, } \\
\text { FAS, FURIN, GRIK2, IL6, INHA, } \\
\text { LCK, RASA1 }\end{array}$ & $\begin{array}{l}\text { BTG3, EPGN, GMNN, } \\
\text { SPHK1 }\end{array}$ & $\begin{array}{l}\text { ANXA1, DSP, GLI1, IL6, LCK, NOG, } \\
\text { ZAP70 }\end{array}$ \\
\hline miR-194 & $\begin{array}{l}\text { COL4A3, FASLG, IFI16, NLRP2, } \\
\text { SRA1, SRGN }\end{array}$ & - & $\begin{array}{l}\text { ACHE, COL4A4, IFI16, IVL, MYH9, } \\
\text { RARA, SRA1 }\end{array}$ \\
\hline $\operatorname{miR}-196 a$ & $\begin{array}{l}\text { CASP8, COL4A3, HBXIP, LCK, } \\
\text { NLRP2, PCBP4, UBE4B }\end{array}$ & PCBP4 & $\begin{array}{l}\text { ACHE, ANXA1, APOB, CSTA, CYFIP1, } \\
\text { DYRK3, ENPP1, LCK, LOR, NFKBIA, } \\
\text { RUNX1, UHRF2 }\end{array}$ \\
\hline miR-212 & $\begin{array}{l}\text { DYRK2, GHRL, IL6, LCK, PEA15, } \\
\text { RASA1 }\end{array}$ & ASNS, GMNN & $\begin{array}{l}\text { ACVR2B, BMPR1A, CAND1, CNTN4, } \\
\text { CSF1, CYFIP1, DOCK7, FARP2, GHRL, } \\
\text { GPR98, IL6, LCK, NRCAM, SART1, SPI1, } \\
\text { TGM3, TNP1 }\end{array}$ \\
\hline $\operatorname{miR}-221^{*}$ & CECR2, GRIK2 & - & $\begin{array}{l}\text { EIF2B3, MOV10L1, RUNX1, SCEL, } \\
\text { TGM1, TNP1 }\end{array}$ \\
\hline $\operatorname{miR}-222^{*}$ & APOH, BNIP1, CD70, NLRP1 & GMNN & $\begin{array}{l}\text { CSTA, CYFIP1, NF2, SPRR1A, TGM3, } \\
\text { TTN, USH2A }\end{array}$ \\
\hline $\operatorname{miR}-362-3 p$ & $\begin{array}{l}\text { ADAMTSL4, ALB, BAX, CADM1, } \\
\text { CARD8, DFFB, DLC1, DYNLL1, } \\
\text { HSPA5, NRG1, RELA, UBE4B }\end{array}$ & $\begin{array}{l}\text { CDKN2D, GTPBP4, } \\
\text { NEK6 }\end{array}$ & $\begin{array}{l}\text { BAX, BMP6, CHD7, DTX1, DYRK3, } \\
\text { IQCB1, KRT19, MOV10L1, NCOA6, NF2, } \\
\text { NRG1, SART1, TNFSF11 }\end{array}$ \\
\hline
\end{tabular}


Table II. Continued.

\begin{tabular}{|c|c|c|c|}
\hline \multirow[b]{2}{*}{ miRNA } & \multicolumn{3}{|c|}{ Biological functions and related genes } \\
\hline & Apoptosis & Cell cycle & Differentiation \\
\hline $\operatorname{miR}-373$ & $\begin{array}{l}\text { BFAR, BNIP1, BNIPL, BRCA1, } \\
\text { PPP3R1, RELA }\end{array}$ & $\begin{array}{l}\text { ALOX15B, CDKN2D, } \\
\text { GTPBP4 }\end{array}$ & CD36, CHRNB1, DSP, SCIN, TCFL5 \\
\hline $\operatorname{miR}-374 b$ & CCL2, IL6 & AFAP1L2 & $\begin{array}{l}\text { ANXA1, CHD7, CHRNA1, IL6, NCOA6, } \\
\text { NOG, TPM1, UHRF2 }\end{array}$ \\
\hline $\operatorname{miR}-424^{*}$ & $\begin{array}{l}\text { CCL2, DNAJB6, GSK3B, HBXIP, } \\
\text { NLRP2, PCSK6 }\end{array}$ & ALOX15B & BTG1, GLI1 \\
\hline $\operatorname{miR}-449 a$ & $\begin{array}{l}\text { AKT1, BAX, BID, DEDD2, DLC1, } \\
\text { HSPD1, TRAF2 }\end{array}$ & CDC23, DBC1, GMNN & $\begin{array}{l}\text { BAX, C1QC, CALCA, EIF2B5, GLMN, } \\
\text { GPR98, MOV10L1, NFKBIA, PPARG, } \\
\text { TGM1, UHRF2, ZAP70 }\end{array}$ \\
\hline $\operatorname{miR}-487 b$ & SRA1, TBX5, TNFRSF10B & $\begin{array}{l}\text { ATR, GTPBP4, HEXIM1, } \\
\text { NEK6, ZW10 }\end{array}$ & $\begin{array}{l}\text { ANXA1, GPR98, HILS1, IVL, PTPRM, } \\
\text { SERPINF1, SRA1, TBX5, TTN }\end{array}$ \\
\hline $\operatorname{miR}-508-3 p$ & LGALS12 & EPGN & ANXA1, MAPK9, SCEL, SYCP3 \\
\hline $\operatorname{miR}-520 d-3 p$ & $\begin{array}{l}\text { BAD, BCL6, BNIP1, BNIPL, BRE, } \\
\text { CASP10, CDKN2A, ERCC3, } \\
\text { FASLG, HBXIP, P2RX4, PCSK6, } \\
\text { TCF7L2, TNFRSF10B }\end{array}$ & $\begin{array}{l}\text { CDC23, CDKN2A, } \\
\text { DBC1, GTPBP4, IL8 }\end{array}$ & $\begin{array}{l}\text { BMPR1A, CD36, CSTA, DSP, ERCC3, } \\
\text { GLMN, MAPK12, PPARG, SCIN, SYCP3, } \\
\text { TCF7L2 }\end{array}$ \\
\hline $\operatorname{miR}-526 b$ & $\begin{array}{l}\text { AIFM3, BAD, BNIPL, BRCA1, } \\
\text { CARD8, ERCC3, F2R, LCK, } \\
\text { PPP3CC }\end{array}$ & - & $\begin{array}{l}\text { ACTA1, CALCA, DOCK } 7, \text { ERCC3, LCK, } \\
\text { MYH9, NF2 }\end{array}$ \\
\hline $\operatorname{miR}-548 c-5 p$ & $\begin{array}{l}\text { ALB, APOH, BAX, CCL2, CHEK2, } \\
\text { DYNLL1, GSK3B, IL6, SON }\end{array}$ & BMP2, BTG3, GTPBP4 & $\begin{array}{l}\text { ALS2, APOB, BAX, BMP2, CALCA, } \\
\text { CHD7, GPR98, IL6, NFKBIA, OBSL1, } \\
\text { PPARG, SLIT2 }\end{array}$ \\
\hline $\operatorname{miR}-557$ & $\begin{array}{l}\text { ALB, BCL6, DLC1, GRIK2, } \\
\text { HTATIP2, LGALS12, NLRP3, SFN, } \\
\text { SRA1, TCF7L2 }\end{array}$ & BMP4, CDKN3, HEXIM2 & BMP4, CCM2, SRA1, TCF7L2, TNFSF11 \\
\hline $\operatorname{miR}-593$ & CD27, DYNLL1, RYR2, STK4 & - & $\begin{array}{l}\text { BMPR1B, CCM2, CHRNA1, DYRK3, } \\
\text { HOXA7, LST1, NKX2-5, TCAP, TCFL5 }\end{array}$ \\
\hline $\operatorname{miR}-625$ & $\begin{array}{l}\text { AKT1, BAD, BAX, CIDEC, } \\
\text { COL4A3, ERCC } 3 \text {, INHBA, NLRP1, } \\
\text { NLRP3 }\end{array}$ & EGF, INHBA, SPHK1 & $\begin{array}{l}\text { BAX, C1QC, CALCA, EIF2B5, ERCC3, } \\
\text { EVPL, INHBA, KCNIP2, MEN1, NF2, } \\
\text { UPK2 }\end{array}$ \\
\hline $\operatorname{miR}-652$ & GRIK2, TCF7L2, TERF1 & BTG3, NEK11 & CALR, NOTCH1, RPS19, TCF7L2 \\
\hline $\operatorname{miR}-877^{*}$ & $\begin{array}{l}\text { BAX, BNIP1, CECR2, GRIK2, } \\
\text { PEA15 }\end{array}$ & BRSK1 & APOB, BAX, CHRNA1, NKX2-5, RUNX1 \\
\hline $\operatorname{miR}-921$ & $\begin{array}{l}\text { BAD, CASP10, FADD, GDNF, } \\
\text { IGF1, TP53, UBE4B }\end{array}$ & $\begin{array}{l}\text { FOXC1, IGF1, NUSAP1, } \\
\text { TCF3 }\end{array}$ & $\begin{array}{l}\text { ADAM9, ERCC2, EVPL, GDNF, GPR98, } \\
\text { HOXA7, IGF1, JAG2, RUNX1, TCF3, } \\
\text { UPK1B }\end{array}$ \\
\hline
\end{tabular}


Table III. Predicted p53 binding sites to upstream regions of miRNAs.

\begin{tabular}{|c|c|c|c|c|c|}
\hline \multirow{3}{*}{$\frac{\text { miRNA }}{\text { miR-7-1 }}$} & \multicolumn{5}{|c|}{ p53-binding site } \\
\hline & \multirow{2}{*}{$\frac{\text { Distance }(\mathrm{kb})^{\mathrm{a}}}{33.7}$} & \multirow{2}{*}{$\frac{\text { Score }(\% \max )^{\mathrm{b}}}{98.43}$} & \multicolumn{3}{|c|}{ Sequences } \\
\hline & & & TGACAAGCTC & . & AGGCATGTTC \\
\hline & 8.0 & 98.43 & AAACATGCCT & AAGATTAG & CAACAAGTCT \\
\hline & 31.2 & 86.47 & TAACATGCCA & TGCAGCTAAA & AGCCAAGTTT \\
\hline \multirow[t]{3}{*}{ miR-9 } & 34.2 & 93.55 & TGGCATGTTA & GT & AAACATGTTT \\
\hline & 27.1 & 91.71 & ACACAAGTTT & $\mathrm{T}$ & CAGCATGCCC \\
\hline & 20.5 & 89.86 & AGGCATGTTT & GCCAGAGTTTGGG & AGACCTGCCC \\
\hline \multirow[t]{3}{*}{ miR-22 } & 46.7 & 100 & AAGCTTGTTT & TA & AAACAAGTTT \\
\hline & 46.7 & 93.48 & ACACAAGCTT & GTTTTA & AAACAAGTTT \\
\hline & 8.2 & 88.52 & AAACTTGTAT & GGAGCATTACCAG & GAGCTAGTCA \\
\hline \multirow[t]{3}{*}{ miR-29b-1 } & 44.4 & 92.75 & AGTCTAGTTC & ATCAG & AAACAAGTTT \\
\hline & 2.5 & 90.75 & AGGCATGTGC & CACCACGCC & TGGCTAGTTT \\
\hline & 28.0 & 89 & AAACTAGCTG & GGCGTCATG & GCACATGCCT \\
\hline \multirow[t]{3}{*}{ miR-30c } & 28.0 & 86.14 & TAGCTAGATC & TTCGGGATA & AAACTTGCTG \\
\hline & 26.5 & 86.14 & AAACATGTCT & TCCCAGGATGGTT & CAACTAGTAG \\
\hline & 15.7 & 86.14 & TAGCATGTCT & GTTG & AAGCATGTGA \\
\hline \multirow[t]{3}{*}{ miR-32 } & 31.6 & 98.12 & AAGCAAGTTC & . & CAACTTGCTT \\
\hline & 48.7 & 93.55 & TGGCTTGCTG & CTATCTGGGAGA & GGGCTAGCTT \\
\hline & 6.1 & 91.71 & TTACTAGCTT & TGTGCCTTT & GGGCAAGTTT \\
\hline \multirow[t]{3}{*}{ miR-100 } & 7.3 & 91.88 & ACACAAGTCT & CCTTAG & CAACTTGTCT \\
\hline & 23.5 & 84.83 & AGGCAAGCTT & GGGTCCTGGCTTA & GACCCTGCCT \\
\hline & 18.9 & 84.33 & AAACTAGTCA & TTCAGTAG & ATACTTGTTG \\
\hline \multirow[t]{3}{*}{ miR-132 } & 17.9 & 92.75 & GAGCAAGTTT & GAG & AGCCTTGTCT \\
\hline & 16.9 & 88.68 & AAACAAGCTT & ССТCTTTG & AAGCCTGTTT \\
\hline & 21.0 & 88.17 & GAGCATGCCT & GGCAACAGAA & GAGCTAGACG \\
\hline \multirow[t]{3}{*}{ miR-141 } & 1.6 & 89.11 & ATACAAGCCG & CAGCTGCACAA & AGGCAAGTCC \\
\hline & 9.5 & 89 & TACCTAGCTC & $\mathrm{T}$ & CAGCATGTTT \\
\hline & 25.1 & 86.58 & GGGCCAGCCC & TGCGGCC & TGACATGTCC \\
\hline \multirow[t]{3}{*}{ miR-153 } & 46.7 & 92.36 & AGGCTTGTTT & TATTTATTGTTAT & TTGCTTGCTT \\
\hline & 46.7 & 88.05 & AGCCTTGCTG & $\mathrm{AG}$ & AGGCTTGTTT \\
\hline & 43.0 & 88.05 & GGTCTTGCTA & TGTTTCCC & AGGCTAGTCT \\
\hline \multirow[t]{3}{*}{ miR-194 } & 2.8 & 92.37 & GAGCAAGTCT & CACTAATATTTAGA & GAGCAAGACT \\
\hline & 43.2 & 86.47 & TAGCATGCCG & ACAGTACC & AGCCTAGCCT \\
\hline & 26.9 & 85.95 & AAACAAGCAA & AGAGAGGT & TAACTTGCCC \\
\hline \multirow[t]{3}{*}{ miR-196a } & 38.7 & 89.22 & TGGCTTGCCC & TAAGTTC & CGACAAGTGC \\
\hline & 33.3 & 87.62 & ATACTTGCTT & CACCCAT & GTACAAGTTC \\
\hline & 28.1 & 86.05 & TGGCCAGCTC & TCTGGTCTTGT & TAACAAGCCT \\
\hline \multirow[t]{3}{*}{ miR-212 } & 17.6 & 92.76 & GAGCAAGTTT & GAG & AGCCTTGTCT \\
\hline & 49.8 & 89.09 & AAACTAGCTG & GGTATGGTG & GCACTTGCCT \\
\hline & 16.5 & 88.72 & AAACAAGCTT & ССТCTTTG & AAGCCTGTTT \\
\hline \multirow[t]{3}{*}{$\operatorname{miR}-221$} & 26.5 & 90.68 & CAGCAAGTCT & GACTCCAG & AAGCTAGACT \\
\hline & 4.7 & 90 & AAGCTTGTTT & TAGGATCTTGTGC & CAACCAGTCC \\
\hline & 13.3 & 87.05 & GGGCAAGTTC & TGTCATCTGGGAGT & TTACATGTTA \\
\hline
\end{tabular}


Table III. Continued.

\begin{tabular}{|c|c|c|c|c|c|}
\hline \multirow{3}{*}{$\frac{\text { miRNA }}{\text { miR-222 }}$} & \multicolumn{5}{|c|}{ p53-binding site } \\
\hline & \multirow{2}{*}{$\frac{\text { Distance }(\mathrm{kb})^{\mathrm{a}}}{25.6}$} & \multirow{2}{*}{$\frac{\text { Score }(\% \max )^{\mathrm{b}}}{90.68}$} & \multicolumn{3}{|c|}{ Sequences } \\
\hline & & & CAGCAAGTCT & GACTCCAG & AAGCTAGACT \\
\hline & 3.9 & 89.99 & AAGCTTGTTT & TAGGATCTTGTGC & CAACCAGTCC \\
\hline & 12.5 & 87.05 & GGGCAAGTTC & TGTCATCTGGGAGT & TTACATGTTA \\
\hline \multirow[t]{3}{*}{ miR-362-3p } & 31.1 & 89.23 & GGGCTAGCCA & GCAG & CAGCAAGCCG \\
\hline & 49.5 & 85.81 & ACTCTTGTTC & CTTGATC & AAGCATGTCC \\
\hline & 15.2 & 85.4 & GGGCCAGTCT & GGCCCAGTA & ACACAAGTCT \\
\hline \multirow[t]{3}{*}{$\operatorname{miR}-373$} & 7.5 & 93.54 & AGACAAGCTG & GAGTGCAA & TGGCATGTTC \\
\hline & 0.1 & 90.71 & AAGCAAGTTT & ATTGCTGACATT & TAACATGTGC \\
\hline & 49.2 & 90.68 & TGGCATGTCC & & AACCTAGCCC \\
\hline \multirow[t]{3}{*}{$\operatorname{miR}-374 b$} & 31.8 & 92.65 & GAGCAAGACC & CAAGTTC & AGGCAAGCTT \\
\hline & 36.6 & 92.16 & GAACTAGTTT & $\mathrm{CCCT}$ & GGACCTGCCC \\
\hline & 31.8 & 86.11 & ACCCAAGTTC & . & AGGCAAGCTT \\
\hline \multirow[t]{3}{*}{$\operatorname{miR}-424$} & 42.3 & 89.64 & CAACCTGTTT & AGCCTCTCACACAT & AAGCTTGCCT \\
\hline & 12.3 & 87.69 & AAACAAGTTA & $\mathrm{CA}$ & GGACTTGACT \\
\hline & 0.9 & 86.01 & GGGCATGCAT & $\mathrm{G}$ & TGACTTGTTG \\
\hline \multirow[t]{3}{*}{ miR-449a } & 36.3 & 89.68 & CTGCATGTTC & TCCC & CAGCAAGCCT \\
\hline & 9.6 & 87.08 & CTACATGTCT & TGCT & GGACATGCTA \\
\hline & 46.4 & 86.16 & GTGCTTGCCT & CATG & AAGCATGCAT \\
\hline \multirow[t]{3}{*}{ miR-487b } & 8.7 & 93.53 & AGACTTGCTT & $\mathrm{CCT}$ & CAACAAGCTG \\
\hline & 33.8 & 93.47 & ATACTTGTCC & TTCAGTTCTAG & AAACTTGTCT \\
\hline & 37.6 & 92.6 & GGGCTTGCCC & CCAGCCCTAG & AGCCTTGCCT \\
\hline \multirow[t]{3}{*}{$\operatorname{miR}-508-3 p$} & 33.6 & 97.97 & GGGCATGTTC & TACTA & TGACTTGTCC \\
\hline & 26.6 & 97.97 & TAGCATGCTC & AAAAC & AAACTTGTTC \\
\hline & 48.3 & 92.57 & AAACATGCTC & TAAAC & AACCTTGCTC \\
\hline \multirow[t]{3}{*}{ miR-520d-3p } & 21.6 & 88.17 & GATCTTGCTA & ATACATTTGCA & GAGCATGCTT \\
\hline & 29.8 & 86.1 & CACCATGTTG & $\mathrm{CCC}$ & AGGCTTGTCT \\
\hline & 18.1 & 86.1 & CACCATGTTG & $\mathrm{CCC}$ & AGGCTAGTCT \\
\hline \multirow[t]{3}{*}{$\operatorname{miR}-526 \mathrm{~b}$} & 48.6 & 95.42 & GAACTTGTTC & TATAT & AAACATGTTA \\
\hline & 27.4 & 90.83 & AGGCAAGTCA & CAACACAGCGAAAG & AAGCAAGCTG \\
\hline & 24.9 & 90.83 & AGGCAAGTCA & CAACACAATGAAAG & AAGCAAGCTG \\
\hline \multirow[t]{3}{*}{$\operatorname{miR}-548 c-5 p$} & 49.7 & 98.18 & CAACTTGTCC & AAGTCTATG & AAGCTTGTTT \\
\hline & 36.8 & 90.74 & AGGCATGTGC & . & CAGCAAGCCT \\
\hline & 11.1 & 89 & AAACAAGCTC & CTATGCTAA & ATACTAGCCA \\
\hline \multirow[t]{3}{*}{ miR-557 } & 44.3 & 100 & AAACAAGCTT & AGAGAGGTTAAG & AAACTTGTTT \\
\hline & 31.5 & 86.2 & GATCTTGCTG & AAGATGAGCTTTT & CAGCTTGCTT \\
\hline & 14.2 & 85.28 & GGTCTAGATT & GAGTGTAAA & GAACTTGCCT \\
\hline \multirow[t]{3}{*}{ miR-593 } & 29.5 & 98.18 & GGACAAGCTC & CCATTCTGG & CAGCTTGTCC \\
\hline & 9.3 & 91.74 & TAGCATGTTT & GATAAAGCT & TGGCAAGTTA \\
\hline & 40.1 & 90.77 & TAACATGACC & ACCTTG & AAACTTGTTC \\
\hline \multirow[t]{3}{*}{ miR-625 } & 3.1 & 91.61 & TAACTTGTTT & GT & TGACAAGTTG \\
\hline & 3.0 & 91.61 & CAACTTGCTT & ACAGTGCCAA & CAGCATGTTG \\
\hline & 3.1 & 90.7 & AATCTTGCCC & $\mathrm{T}$ & TAACTTGTTT \\
\hline
\end{tabular}


Table III. Continued.

\begin{tabular}{|c|c|c|c|c|c|}
\hline \multirow[b]{2}{*}{ miRNA } & \multicolumn{5}{|c|}{ p53-binding site } \\
\hline & Distance $(\mathrm{kb})^{\mathrm{a}}$ & Score $(\% \max )^{\mathrm{b}}$ & & Sequences & \\
\hline \multirow[t]{3}{*}{ miR-652 } & 25.5 & 91.59 & GAGCTAGTCC & TCT & TTGCTAGCCC \\
\hline & 37.6 & 90.71 & AGGCATGCCC & . & CACCATGCCC \\
\hline & 10.4 & 88.75 & TAGCTTGCTT & TACTCTC & TGGCATGTAT \\
\hline \multirow[t]{3}{*}{ miR-877 } & 30.4 & 92.42 & GAACATGTGT & $\mathrm{GC}$ & AAACATGCCT \\
\hline & 7.8 & 91.31 & CACCATGTTC & $\mathrm{ACC}$ & AGACTAGTCT \\
\hline & 6.6 & 88.79 & CAGCCTGCTT & GGATTGCTCTTGG & AAACATGTTT \\
\hline \multirow[t]{3}{*}{ miR-921 } & 30.9 & 92.43 & AGGCTAGCTT & . & GAGCTTGATC \\
\hline & 31.6 & 91.22 & TATCAAGCTC & . & AAGCTAGTCT \\
\hline & 29.6 & 87.34 & ATGCTAGTCC & . & TAGCAAGCTA \\
\hline
\end{tabular}

${ }^{\mathrm{a} D i s t a n c e}$ of $\mathrm{p} 53$ binding site upstream from pre-miRNA sequence. ${ }^{\mathrm{b}} \mathrm{Calculated}$ scores are presented as percentages.

Next, we predicted the target genes for those miRNAs showing a $>5$-fold change and identified genes related to apoptosis, cell cycle, and differentiation, which are the biological processes observed in tumor cells treated with HDACIs (Table II). miRNA targets were predicted by miRBase Targets Version 5, and Gene Ontology was used to identify genes related to the three biological processes mentioned above. Each miRNA was predicted to have a range from hundreds to thousands of targets. Genes related to apoptosis, cell cycle, and cellular arrest and differentiation had 172, 69, and 192 miRNAs, respectively, associated with them. These genes are listed in Table II. As reported by John et al (39), each miRNA targets several mRNAs, and we found 71, 51, and 19 genes, respectively, targeted by more than two miRNAs in each of the three functional groups.

Potential p53 binding sites for the miRNAs in Table II were analyzed by using the p53MH program. We collected $50 \mathrm{~kb}$ of upstream sequence from precursor miRNAs using the UCSC human genome database. Long upstream sequences were obtained because p53 is known to bind some miRNAs $30 \mathrm{~kb}$ upstream (40). Table III lists the top three putative p52 binding sites for each of the 31 miRNAs. Most of the predicted sites had scores $>5 \%$, and two miRNAs, miR-22* and -557 , had sites scored as $100 \%$.

In conclusion, we identified several miRNAs whose expression was affected by SAHA and p53. Many of the miRNAs were dramatically changed by these factors and predicted to target several mRNAs. Although further studies are needed to prove these predictions, they give us important clues in understanding the function and regulation of miRNAs.

\section{Acknowledgements}

We are grateful to all other members in our research group for their support and advice in regards to this study. This work was supported by the Nuclear Research and Development Program of National Research Foundation of Korea funded by the Ministry of Education, Science, and Technology (grant codes: M20706000020-08M060002000 and 20090078237) and the Ministry of Knowledge Economy (R-2006-1-043) of the Republic of Korea.

\section{References}

1. Jenuwein T and Allis CD: Translating the histone code. Science 293: 1074-1080, 2001.

2. Turner BM: Cellular memory and the histone code. Cell 111: 285-291, 2002.

3. Verdone L, Caserta M and Di Mauro E: Role of histone acetylation in the control of gene expression. Biochem Cell Biol 83: 344-353, 2005.

4. Phillips AC and Vousden KH: Acetyltransferases and tumour suppression. Breast Cancer Res 2: 244-246, 2000.

5. Minucci S, Nervi C, Lo Coco F and Pelicci PG: Histone deacetylases: a common molecular target for differentiation treatment of acute myeloid leukemias? Oncogene 20: 3110-3115, 2001.

6. Jones PA and Baylin SB: The fundamental role of epigenetic events in cancer. Nat Rev Genet 3: 415-428, 2002.

7. Yang XJ and Seto E: HATs and HDACs: from structure, function and regulation to novel strategies for therapy and prevention. Oncogene 26: 5310-5318, 2007.

8. Drummond DC, Noble CO, Kirpotin DB, Guo Z, Scott GK and Benz CC: Clinical development of histone deacetylase inhibitors as anticancer agents. Annu Rev Pharmacol Toxicol 45: 495-528, 2005.

9. Martínez-Iglesias O, Ruiz-Llorente L, Sánchez-Martínez R, García L, Zambrano A and Aranda A: Histone deacetylase inhibitors: mechanism of action and therapeutic use in cancer. Clin Transl Oncol 10: 395-398, 2008.

10. Bolden JE, Peart MJ and Johnstone RW: Anticancer activities of histone deacetylase inhibitors. Nat Rev Drug Discov 5: 769-784, 2006.

11. Yoshida M, Kijima M, Akita M and Beppu T: Potent and specific inhibition of mammalian histone deacetylase both in vivo and in vitro by trichostatin A. J Biol Chem 265: 17174-17179, 1990.

12. Kijima M, Yoshida M, Sugita K, Horinouchi S and Beppu T: Trapoxin, an antitumor cyclic tetrapeptide, is an irreversible inhibitor of mammalian histone deacetylase. J Biol Chem 268: 22429-22435, 1993.

13. Marks PA, Richon VM and Rifkind RA: Histone deacetylase inhibitors: inducers of differentiation or apoptosis of transformed cells. J Natl Cancer Inst 92: 1210-1216, 2000. 
14. Terui T, Murakami K, Takimoto R, et al: Induction of PIG3 and NOXA through acetylation of p53 at 320 and 373 lysine residues as a mechanism for apoptotic cell death by histone deacetylase inhibitors. Cancer Res 63: 8948-8954, 2003.

15. Hsi LC, Xi X, Lotan R, Shureiqi I and Lippman SM: The histone deacetylase inhibitor suberoylanilide hydroxamic acid induces apoptosis via induction of 15-lipoxygenase-1 in colorectal cancer cells. Cancer Res 64: 8778-8781, 2004.

16. Eyüpoglu IY, Hahnen E and Buslei R, et al: Suberoylanilide hydroxamic acid (SAHA) has potent anti-glioma properties in vitro, ex vivo and in vivo. J Neurochem 93: 992-999, 2005.

17. Gu W and Roeder RG: Activation of $\mathrm{p} 53$ sequence-specific DNA binding by acetylation of the p53 C-terminal domain. Cell 90: 595-606, 1997.

18. Imhof A, Yang XJ, Ogryzko VV, Nakatani Y, Wolffe AP and $\mathrm{Ge} \mathrm{H}$ : Acetylation of general transcription factors by histone acetyltransferases. Curr Biol 7: 689-692, 1997.

19. Boyes J, Byfield P, Nakatani Y and Ogryzko V: Regulation of activity of the transcription factor GATA-1 by acetylation. Nature 396: 594-598, 1998.

20. Wen YD, Cress WD, Roy AL and Seto E: Histone deacetylase 3 binds to and regulates the multifunctional transcription factor TFII-I. J Biol Chem 278: 1841-1847, 2003.

21. Matsumura T, Suzuki T, Aizawa K, Munemasa Y, Muto S, Horikoshi $\mathrm{M}$ and Nagai R: The deacetylase HDAC1 negatively regulates the cardiovascular transcription factor Krüppellike factor 5 through direct interaction. J Biol Chem 280 : 12123-12129, 2005

22. Chuang HC, Chang CW, Chang GD, Yao TP and Chen H: Histone deacetylase 3 binds to and regulates the GCMa transcription factor. Nucleic Acids Res 34: 1459-1469, 2006.

23. Johnstone RW and Lich JD: Histone deacelylase inhibitors in cancer therapy: is transcription the primary target? Cancer Cell 4: 13-18, 2003.

24. Bernstein E, Caudy AA, Hammond SM and Hannon GJ: Role for a bidentate ribonuclease in the initiation step of RNA interference. Nature 409: 363-366, 2001.

25. Lee Y, Jeon K, Lee JT, Kim S and Kim VN: MicroRNA maturation: stepwise processing and subcellular localization. EMBO J 21: 4663-4670, 2002.

26. Lee Y, Kim M, Han J, et al: MicroRNA genes are transcribed by RNA polymerase II. EMBO J 23: 4051-4060, 2004.
27. Denli AM, Tops BB, Plasterk RH, Ketting RF and Hannon GJ: Processing of primary microRNAs by the Microprocessor complex. Nature 432: 231-235, 2004.

28. Gregory RI, Chendrimada TP, Cooch N and Shiekhattar R: Human RISC couples microRNA biogenesis and posttranscriptional gene silencing. Cell 123: 631-640, 2005.

29. Lee RC, Feinbaum RL and Ambros V: The C. elegans heterochronic gene lin-4 encodes small RNAs with antisense complementarity to lin-14. Cell 75: 843-854, 1993.

30. Alvarez-Garcia I and Miska EA: MicroRNA functions in animal development and human disease. Development 132: 4653-4662, 2005.

31. Kent OA and Mendell JT: A small piece in the cancer puzzle: microRNAs as tumor suppressors and oncogenes. Oncogene 2: 6188-6196, 2006.

32. Bueno MJ, De Castro IP and Malumbres M: Control of cell proliferation pathways by microRNAs. Cell Cycle 7: 3143-3148, 2008.

33. Marson A, Levine SS, Cole MF, et al: Connecting microRNA genes to the core transcriptional regulatory circuitry of embryonic stem cells. Cell 134: 521-533, 2008.

34. Lu J, Getz G, Miska EA, et al: MicroRNA expression profiles classify human cancers. Nature 435: 834-838, 2005.

35. Sassen S, Miska EA and Caldas C: MicroRNA: implications for cancer. Virchows Arch 452: 1-10, 2008.

36. Butler LM, Zhou X, Xu WS, et al: The histone deacetylase inhibitor SAHA arrests cancer cell growth, up-regulates thioredoxin-binding protein-2, and down-regulates thioredoxin. Proc Natl Acad Sci USA 99: 11700-11705, 2002.

37. Hoh J, Jin S, Parrado T, Edington J, Levine AJ and Ott J: The p53 $\mathrm{MH}$ algorithm and its application in detecting p53responsive genes. Proc Natl Acad Sci USA 99: 8467-8472, 2002.

38. Griffiths-Jones S, Saini HK, van Dongen S and Enright AJ: miRBase: tools for microRNA genomics. Nucleic Acids Res 36: D154-D158, 2008.

39. John B, Enright AJ, Aravin A, Tuschi T, Sander C and Marks D: Human MicroRNA targets. PLoS Biol. 2: e363, 2004.

40. Chang TC, Wentzel EA, Kent OA, et al: Transactivation of miR-34a by p53 broadly influences gene expression and promotes apoptosis. Mol Cell 26: 745-752, 2007. 